\title{
Conhecimento do cirurgião dentista sobre filmes radiográficos interferindo na otimização do processamento radiográfico
}

\author{
Knowledge of the clinical dentist about radiographic film interfering in processing optimization \\ Conocimiento del dentista clínico sobre la interferencia de la película radiográfica \\ en la optimización del procesamiento
}

\author{
Dayane Dias ARAÚJO ${ }^{1}$ \\ Douglas Dias ARAÚJO1 \\ Marina Santana GAZOLLA ${ }^{2}$ \\ Matheus Henrique Lopes DOMINGUETE \\ Luiz Augusto Maia de OLIVEIRA ${ }^{2}$ \\ Kamila Prado Pereira GRACIANO ${ }^{3}$ \\ Cátia Marisa Gazolla de OLIVEIRA ${ }^{4}$
}

\begin{abstract}
${ }^{1}$ Acadêmica do Curso de Odontologia, Universidade Vale do Rio Verde UninCor, 37410 Três Corações-MG, Brasil
${ }^{2}$ Professor (a) do Curso de Odontologia, Departamento de Diagnóstico e Cirurgia Oral, Universidade Vale do Rio Verde UninCor 37410 Três Corações-MG, Brasil
\end{abstract}

${ }^{3}$ Mestre em Odontologia, Departamento de Diagnóstico e Cirurgia Oral, Universidade Vale do Rio Verde UninCor, 37410 Três Coraçães-MG, Brasil

${ }^{4}$ Professora Orientadora, Departamento de Diagnóstico e Cirurgia Oral, Universidade Vale do Rio Verde UninCor, 37410 Três Corações-MG, Brasil

\section{Resumo}

Objetivo: O objetivo deste trabalho foi avaliar o conhecimento sobre filmes radiográficos na odontologia e suas características, para definir o que melhor se enquadra nos seus procedimentos. Metodologia: Foi elaborado um questionário sobre filmes radiográficos quanto à seleção na aquisição, sensibilidade dos filmes e quais estão sendo utilizados. O questionário foi respondido por 50 cirurgiões-dentistas escolhidos aleatoriamente, excluindo-se os radiologistas. Resultados: Foi observado que 35 (70\%) dos participantes disseram saber o que é sensibilidade e $29(58 \%)$ afirmaram que compram o filme de acordo com a sensibilidade. Ao verificar o conhecimento real do assunto, menos da metade das questões foram respondidas corretamente. Outro fato importante, é que embora 35 (70\%) dos participantes soubessem o que é sensibilidade, somente $16(32 \%)$ deles estão usando os filmes mais sensíveis existentes no mercado. Observou-se também que 17 (34\%) dos profissionais participantes têm entre vinte e trinta anos, sendo que 27 (54\%) se formaram a partir do ano 2000. Conclusão: Concluiu-se que, apesar dos profissionais afirmarem saber o que é sensibilidade e escolher o filme por este motivo, na pratica isso não acontece. Os resultados destacam que é necessário um esclarecimento maior dos profissionais ao que se refere ao emprego das radiações

Descritores: Radiografia Dentária; Filme para Raios X; Conhecimento.

\section{Abstract}

Objective: The aim of this study was to investigate the knowledge of the dentist, about radiographic films in dentistry, in order to define what best fits into your procedures and know how to choose what should be used to bring benefit to the patient. Methods: A questionnaire containing questions about acquisition, knowledge and sensitivity of these films was answered by 50 dentists randomly selected, excluding radiologists. Results: It was observed that $35(70 \%)$ of participants say they know what's sensitivity and 29 (58\%) say they buy the film according to sensitivity. To verify the actual knowledge of the subject, less than half of the questions were answered correctly. Another important fact is that $35(70 \%)$ of the participants know what sensitivity is, only $16(32 \%)$ of them are using the most sensitive films. It was also observed that 17 (34\%) of the professional participants have between twenty and thirty years, and 27 (54\%) were formed after 2000. Conclusion: It is concluded that despite the professional claim to know the film's sensitivity and choose the film for this reason, in practice it doesn't happen. The results highlight that further clarification of professionals with regard to employment of radiation is necessary.

Descriptors: Radiography, Dental; X-Ray Film; Knowledge.

\section{Resumen}

Objetivo: El objetivo de este trabajo fue evaluar el conocimiento sobre películas radiográficas en la Odontología y sus características, para definir lo que mejor se encuadra en sus procedimientos. Metodología: Se ha elaborado un cuestionario sobre películas radiográficas en cuanto a la selección en la adquisición, sensibilidad de las películas y cuáles están siendo utilizados. El cuestionario fue respondido por 50 odontólogos elegidos aleatoriamente, excluyendo a los radiólogos. Resultados: Se observó que 35 (70\%) de los participantes dijeron saber qué es sensibilidad y $29(58 \%)$ afirmaron que compran la película de acuerdo con la sensibilidad. Al verificar el conocimiento real del asunto, menos de la mitad de las preguntas fueron respondidas correctamente. Otro hecho importante, es que aunque 35 (70\%) de los participantes supieran lo que es sensibilidad, sólo $16(32 \%)$ de ellos están usando las películas más sensibles existentes en el mercado. Se observó también que 17 (34\%) de los profesionales participantes tienen entre veinte y treinta años, siendo que 27 (54\%) se formaron a partir del año 2000. Conclusión: Se concluyó que, a pesar de que los profesionales afirman saber lo que es la sensibilidad y elegir la película por este motivo, en la práctica no sucede. Los resultados señalan que es necesario un mayor aclaramiento de los profesionales en lo que se refiere al empleo de las radiaciones

Descriptores: Radiografía Dental; Película para Rayos X; Conocimiento.

\section{INTRODUÇÃO}

A avaliação de exames radiográficos é o meio mais utilizado e indispensável para o diagnóstico e planejamento, durante e após o tratamento de um caso em Odontologia, entretanto, a radiação $X$ utilizada nesses exames é capaz de provocar efeitos deletérios aos seres vivos ${ }^{1-4}$.

Com o passar dos anos, muitas pesquisas foram implementadas não só com o objetivo de aperfeiçoar os aparelhos emissores de raios X, como também desenvolver melhores filmes radiográficos, em especial aperfeiçoando a sensibilidade dos mesmos. Com isso reduziram-se os efeitos biológicos prejudiciais da utilização dos raios $X$, tanto para o paciente, quanto para o profissional ${ }^{5-7}$.

Há uma busca constante por filmes que necessitem de um menor tempo de exposição aos raios $\mathrm{X}$, sem perda do contraste ou resolução, e que proporcione uma imagem radiográfica adequada para 
um diagnóstico preciso. Atualmente existem no mercado filmes radiográficos com diferentes velocidades, classificados em sensibilidade "D", "E" e "F" $"$-10.

Os filmes mais sensíveis ou de maior velocidade devem ser sempre os preferidos quando da escolha de procedimentos radiográficos, pois minimizam a dose de radiação recebida pelos pacientes, reduzindo-se assim os riscos radio biológicos $^{9-12}$.

$\mathrm{O}$ presente estudo objetivou pesquisar o conhecimento do cirurgião-dentista (CD) sobre filmes radiográficos, com relação aos tipos de filmes existentes no mercado, sua escolha para a utilização e principalmente, o benefício de cada um ao seu paciente.

\section{MATERIAL E MÉTODO}

O presente estudo foi submetido e aprovado pelo Comitê de Ética em Pesquisa da Universidade Vale do Rio Verde de Três Corações-MG, sob parecer $n^{\circ}$ 726.610. A pesquisa foi realizada com cirurgiões-dentistas localizados na cidade de Três Corações, Minas Gerais, Brasil, entre eles, clínicos gerais, especialistas, mestres, doutores e recémformados, excluindo-se os radiologistas.

Os cirurgiões-dentistas foram escolhidos aleatoriamente por meio de de abordagem pessoal, mediante a aceitação do Termo de Consentimento Livre e Esclarecido, devidamente apresentado previamente a pesquisa. Para os entrevistados que decidiram participar do estudo foi apresentado um questionário constituído de 6 perguntas relacionados ao nível do conhecimento dos cirurgiões-dentistas com relação a sensibilidade, aquisição e utilização dos filmes radiográficos mais utilizados. Este questionário continha também 3 questões relacionadas ao perfil do profissional incluindo: idade, ano de formatura e qualificação profissional (Tabela 1).

As informações coletadas a partir dos questionários foram transcritas para um banco de dados (Microsoft Excel ${ }^{\circledR}$ - Microsoft Corporation) e analisados estatisticamente com o suporte do software SPSS ${ }^{2} 20$ for Windows (Statistical Package for Social Sciences). Os dados foram analisados e os resultados foram expressos em porcentagem para a avaliação do conhecimento sobre filmes radiográficos

\section{RESULTADOS}

Foram analisados 50 questionários, os quais se alinhavam aos critérios de inclusão do estudo. Observou-se que a maioria dos entrevistados encontrava-se na faixa etária de 20 a 30 anos de idade (34\%) e que $54 \%$ dos entrevistados se graduaram a partir do ano 2000. Em relação à qualificação profissional, $80 \%$ dos CDs são pós-graduados, com pelo menos o título de especialista (50\%).
Tabela 1. Questionário aplicado

\begin{tabular}{|c|c|}
\hline Idade & \\
\hline \multicolumn{2}{|l|}{ Ano de formatura } \\
\hline Qualificação Profissional & $\begin{array}{l}\text { ( ) Graduação } \\
\text { ( ) Especialização } \\
\text { ( ) Mestrado } \\
\text { ( ) Doutorado }\end{array}$ \\
\hline $\begin{array}{l}\text { Você faz radiografia no seu } \\
\text { consultório? }\end{array}$ & $\begin{array}{l}\text { ( ) Sim } \\
\text { ( ) Não }\end{array}$ \\
\hline \multicolumn{2}{|l|}{$\begin{array}{l}\text { No momento qual filme você } \\
\text { está usando? }\end{array}$} \\
\hline $\begin{array}{l}\text { O que é levado em conta ao } \\
\text { adquirir o filme radiográfico: }\end{array}$ & $\begin{array}{l}\text { ( ) O preço } \\
\text { ( ) A sensibilidade do } \\
\text { filme } \\
\text { ( ) O que é mais vendido } \\
\text { ( ) A marca comercial } \\
\text { ( ) O que o vendedor } \\
\text { afirmar ser o melhor }\end{array}$ \\
\hline $\begin{array}{l}\text { Você sabe o que é sensibilidade } \\
\text { do filme? }\end{array}$ & $\begin{array}{l}\text { ( ) Sim } \\
\text { ( ) Não } \\
\text { ( ) Estou em dúvida }\end{array}$ \\
\hline $\begin{array}{l}\text { Qual filme Radiográfico você } \\
\text { usa? }\end{array}$ & $\begin{array}{l}\text { ( ) Kodak® } \\
\text { ( ) Kodak Ultra® } \\
\text { ( ) E-Speed® } \\
\text { ( ) Kodak Insight® } \\
\text { ( ) Nenhum }\end{array}$ \\
\hline $\begin{array}{l}\text { Quanto a sensibilidade, marque } \\
\text { V ou F: }\end{array}$ & $\begin{array}{l}\text { ( ) A sensibilidade é } \\
\text { determinada pelo tamanho } \\
\text { dos cristais de prata } \\
\text { ( ) Quanto maiores os } \\
\text { cristais, menos radiação } \\
\text { será necessária para a } \\
\text { radiografia } \\
\text { ( ) A ordem crescente de } \\
\text { sensibilidade em relação } \\
\text { aos grupos de filmes é: } \\
\text { Grupo D, E e F } \\
\text { ( ) O grupo D são os } \\
\text { filmes chamados "Ultra } \\
\text { speed" } \\
\text { ( ) O grupo E são os } \\
\text { filmes chamados "Ekta } \\
\text { speed" } \\
\text { ( ) Quanto mais sensível é } \\
\text { o filme, maior definição } \\
\text { será obtida na imagem } \\
\text { radiográfica } \\
\text { ( ) Já existe no mercado } \\
\text { filmes do Grupo F }\end{array}$ \\
\hline
\end{tabular}

A análise dos questionários demonstra que $70 \%$ dos CDs fazem o exame radiográfico no consultório, e que $58 \%$ dos entrevistados, ao realizar a aquisição do filme radiográfico, leva em conta a sensibilidade da película. Observou-se também, que a marca comercial também exerce uma grande influência na escolha, sendo que a marca Kodak ${ }^{\circledR}$, foi a que os CDs relataram ser a que mais adquirida (38\%). Os resultados dos questionários podem ser melhor visualizados na Tabela 2.

Cerca de $70 \%$ dos participantes relatam saber o que é sensibilidade do filme. Entretanto, ao responderem as sete questões sobre conceitos básicos de sensibilidade do filme radiográfico, em nenhuma 
das questões a porcentagem de acertos foi igual ou superior aos $70 \%$. Novamente $70 \% \mathrm{CDs}$ responderam que sabem o que é sensibilidade, mas somente 16 $(32 \%)$ dos profissionais utilizam os filmes mais sensíveis. Os resultados dos questionários estão apresentados na Tabela 3.

Tabela 2. Valores descritivos sobre o perfil profissional dos entrevistados, critérios de seleção e tipo de filme radiográfico utilizado

\begin{tabular}{|c|c|c|c|}
\hline & & $\mathbf{N}$ & $\%$ \\
\hline \multirow[t]{5}{*}{ Idade } & 20 a 29 anos & 17 & $34,0 \%$ \\
\hline & 30 a 39 anos & 16 & $32,0 \%$ \\
\hline & 40 a 49 anos & 10 & $20,0 \%$ \\
\hline & 50 a 60 anos & 6 & $12,0 \%$ \\
\hline & Acima de 60 anos & 1 & $2,0 \%$ \\
\hline \multirow[t]{4}{*}{ Ano de Graduação } & Década de 70 & 2 & $4,0 \%$ \\
\hline & Década de 80 & 10 & $20,0 \%$ \\
\hline & Década de 90 & 11 & $22,0 \%$ \\
\hline & A partir do ano 2000 & 27 & $54,0 \%$ \\
\hline \multirow{4}{*}{$\begin{array}{l}\text { Capacitação } \\
\text { Profissional }\end{array}$} & Graduação & 10 & $20,0 \%$ \\
\hline & Especialização & 25 & $50,0 \%$ \\
\hline & Mestrado & 7 & $14,0 \%$ \\
\hline & Doutorado & 8 & $16,0 \%$ \\
\hline \multirow{2}{*}{$\begin{array}{l}\text { Você realiza exame } \\
\text { radiográfico no } \\
\text { consultório? }\end{array}$} & Sim & 35 & $70,0 \%$ \\
\hline & Não & 15 & $30,0 \%$ \\
\hline \multirow{4}{*}{$\begin{array}{l}\text { O que leva em conta } \\
\text { ao adquirir o filme } \\
\text { radiográfico? }\end{array}$} & Sensibilidade do filme & 29 & $46,0 \%$ \\
\hline & Preço de mercado & 12 & $19,0 \%$ \\
\hline & A marca comercial & 21 & $33,3 \%$ \\
\hline & O que é mais vendido & 1 & $1,6 \%$ \\
\hline \multirow{5}{*}{$\begin{array}{l}\text { Qual filme } \\
\text { radiográfico você } \\
\text { usa? }\end{array}$} & Kodak® & 18 & $36,0 \%$ \\
\hline & Kodak Ultra® & 0 & $0,0 \%$ \\
\hline & E-Speed $\AA$ & 13 & $26,0 \%$ \\
\hline & Kodak Insight $®$ & 3 & $6,0 \%$ \\
\hline & Nenhum & 16 & $32,0 \%$ \\
\hline
\end{tabular}

Tabela 3. Valores referentes às respostas sobre a sensibilidade dos filmes radiográficos

\begin{tabular}{|c|c|c|c|}
\hline & & $\mathbf{N}$ & $\%$ \\
\hline \multirow{3}{*}{$\begin{array}{llr}\text { Você sabe } & \text { o } & \text { que é } \\
\text { sensibilidade } & \text { do } & \text { filme } \\
\text { radiográfico? } & & \end{array}$} & Sim & 35 & $70,0 \%$ \\
\hline & Não & 5 & $10,0 \%$ \\
\hline & Tenho dúvida & 10 & $20,0 \%$ \\
\hline \multirow{2}{*}{$\begin{array}{l}\text { A sensibilidade é } \\
\text { determinada pelo tamanho } \\
\text { dos cristais de prata? }\end{array}$} & Verdadeiro & 34 & $68,0 \%$ \\
\hline & Falso & 16 & $32,0 \%$ \\
\hline \multirow{2}{*}{$\begin{array}{l}\text { Quanto maiores os cristais, } \\
\text { menor radiação r será } \\
\text { necessária para a realização } \\
\text { da radiografia? }\end{array}$} & Verd & 27 & $54,0 \%$ \\
\hline & Falso & 23 & $46,0 \%$ \\
\hline \multirow{2}{*}{$\begin{array}{l}\text { Já existe no mercado filmes } \\
\text { do grupo } F\end{array}$} & Verd & 24 & $48,0 \%$ \\
\hline & Falso & 26 & $52,0 \%$ \\
\hline \multirow{2}{*}{$\begin{array}{l}\text { A ordem crescente de } \\
\text { sensibilidade dos filmes é: } \\
\text { grupo D, E, F? }\end{array}$} & Verdadeiro & 28 & $56,0 \%$ \\
\hline & Falso & 22 & $44,0 \%$ \\
\hline \multirow{2}{*}{$\begin{array}{l}\text { O grupo E são os chamados } \\
\text { "ekta speed"? }\end{array}$} & Verdadeiro & 30 & $60,0 \%$ \\
\hline & \begin{tabular}{|l|} 
Falso \\
\end{tabular} & 20 & $40,0 \%$ \\
\hline \multirow{2}{*}{$\begin{array}{l}\text { O grupo D são os chamados } \\
\text { "ultra speed"? }\end{array}$} & Verdadeiro & 32 & $64,0 \%$ \\
\hline & Falso & 18 & $36,0 \%$ \\
\hline \multirow{2}{*}{$\begin{array}{l}\text { Quanto mais sensível o } \\
\text { filme, maior a definição na } \\
\text { imagem radiográfica }\end{array}$} & Verdadeiro & 36 & $72,0 \%$ \\
\hline & Falso & 14 & $28,0 \%$ \\
\hline
\end{tabular}

\section{DISCUSSÃO}

A maioria dos processos patológicos com que o cirurgião-dentista se vê envolvido localiza-se em tecidos duros e ossos, oferecendo dificuldade a utilização plena dos exames clínicos. Por essa razão, os exames radiográficos são de especial importância na Odontologia e dificilmente podem ser descartados no processo de diagnóstico ${ }^{13}$.

Tosoni et al. ${ }^{14}$ avaliaram 395 cirurgiõesdentistas de diferentes especialidades, tempo de formação profissional e regiões do país, observaram que $99 \%$ dos cirurgiões-dentistas participantes realizam exames radiográficos intrabucais. Esse fato também justifica a alta porcentagem (70\%) encontrada no presente trabalho de entrevistados que realizam exames radiográficos odontológicos.

Miyahara et al. ${ }^{15}$ e Salineiro e Capelozza ${ }^{5}$ observaram em suas pesquisas, que ao realizar a aquisição do filme radiográfico, $55 \%$ a $73 \%$ dos cirurgiões dentistas levam em conta a sensibilidade do mesmo, resultado coincidente com os encontrados na presente pesquisa $(58 \%)$. Contudo, nossos resultados discordam de algumas outras investigações, em que a marca comercial é o primeiro fator levado em conta a aquisição do filme radiográfico $^{4,7}$.

Estudos anteriores observaram que $68 \%$ a $83 \%$ dos cirurgiões-dentistas afirmam saber o que é sensibilidade do filme, assim como em nossa investigação $(70 \%){ }^{5,9}$ Porém, ao analisar em nosso estudo as questões sobre conceitos básicos de sensibilidade do filme radiográfico, apenas 42,9\% foram respondidas corretamente, resultado concordante com os de outros autores, que ressaltaram encontrar baixa porcentagem de acertos em relação ao assunto ${ }^{4,15,16}$.

De acordo com Sheaffer et al. ${ }^{12}$ o filme Insight (sensibilidade $\mathrm{F}$ ) é $25 \%$ mais rápido que o Ekta speed Plus (sensibilidade E) e $60 \%$ mais rápido que o Ultra Speed (sensibilidade D). Considerando-se o princípio de que o paciente deveria ser exposto a mais baixa dose de radiação possível, seria aconselhável que se utilizasse os filmes mais sensíveis para se obter as imagens radiográficas sem prejuízo da qualidade da imagem ${ }^{11}$.

Apesar de poucos estudos terem relatado que os profissionais utilizam os filmes mais sensíveis ${ }^{9}$, existe um consenso na literatura de que os filmes sensibilidade F não são muito utilizados por falta do conhecimento da sua existência ${ }^{6,13,14}$. Esse fato também justifica em nosso estudo, a baixa porcentagem encontrada $(6 \%)$ dos profissionais que utilizam estes filmes mais sensíveis (filmes F).

\section{CONCLUSÃO}

Com o presente estudo, foi concluído que apesar dos profissionais afirmarem saber o que é 
sensibilidade e escolher o filme por este motivo, quando foi levado à prática, o conceito de sensibilidade é equivocado, e os filmes mais sensíveis não estão sendo utilizados pela maioria.

Julga-se necessário um esclarecimento maior dos profissionais no que se refere ao emprego das radiações. As empresas lançam no mercado melhores filmes radiográficos odontológicos e estes devem ser levados ao conhecimento dos profissionais. Deve ser buscado cada vez mais, um rígido controle de qualidade onde os filmes mais rápidos devem ser utilizados para minimizar a dose de exposição à radiação do paciente.

\section{REFERÊNCIAS}

1. Sales MAO, Bonifácio KC, Medeiros AMC, Watanabe PCA, Pardini LC. Aparelho de raios $\mathrm{X}$ odontológico: avaliaçäo densitométrica da radiaçäo de fuga do receptor da imagem. RPG: Rev Pós-Grad. 1997; 4(1):34-8.

2. Woitchchunas GFP, Veeck EB, Holderbaum RM, Souza PHC. Avaliação in vitro da sensibilidade de três filmes radiográficos. Passo Fundo. 2001; 6(2):61-5.

3. Cruz LP, Moraes LC, Kohatsu LI, Moraes MEL, Castilho JCM, Medici Filho E. Grau de conhecimento dos cirurgiões-dentistas sobre os métodos radiográficos de localização. Rev RGO. 2009; 57(3):269-72.

4. Neves FS, Vasconcelos TV, Bastos LC, Góes LA, Freitas DQ. Atitudes dos cirurgiõesdentistas em relação à proteção radiológica, de acordo com a lei brasileira. Rev Odontol Bras Central. 2010; 19(51):301-5.

5. Salineiro S, Capelozza A. Avaliação das condições de radioproteção em cem consultórios odontológicos da cidade de Araçatuba, estado de São Paulo. Rev FOB. 1997; 5(1/2):65-70.

6. Farman TT, Farman AG. Evaluation of a new F speed dental X ray film. The effect of processing solutions and a comparison with D and E speedfilms. Dentomaxillofac Radiol. 2000; 29(1):41-5.

7. Lemke F, Tavano O, Mezadri AC. Verificação das condições de exposição e processamento de filmes radiográficos em consultórios odontológicos. RPG: Rev Pós-Grad. 2006; 13(2):175-80.

8. Watanabe PCA. Filtração adicional da radiação $\mathrm{X}$ em odontologia. Estudo comparativo entre filtros de alumínio e cobre [tese]. São Paulo: Universidade de São Paulo; 1999.

9. Costa C, David AF, David SMN, Matsui RH, Castilho JCM, Varoli FP. Estudo das densidades base e velamento obtidas de filmes radiográficos em diferentes condições de processamento. Ciênc Odontol Bras. 2005; 8(1):90-6
10. Pardini LC, Watanabe PCA, Monteiro S. Filme radiográfico intraoral: controle de qualidade. Rev Bras Teleodonto. 2006; 2(3):11-6.

11. Tamburus JR. Quality of radiographic images: laboratory evaluation of intraoral films, filters, collimators, and radiation exposure. Rev Odontol Univ São Paulo. 1997; 11(3):161-67.

12. Sheaffer JC, Eleazer PD, Scheetz JP, Clark SJ, Farman AG. A comparison of D-, E-, and Fspeed conventional intraoral radiographic films in endodontic measurement. Oral Surg Oral Med Oral Pathol Oral Radiol Endod. 2002; 93(3):337-40.

13. Bernstein DI, Clark SJ, Scheetz JP, Farman AG, Rosenson B. Perceived quality of radiographic images after rapid processing of D- and F-speed direct-exposure intraoral x-ray films. Oral Surg Oral Med Oral Pathol Oral Radiol Endod. 2003; 96(4):486-91.

14. Tosoni GM, Campos DM, Silva MR. Frequência de cirurgiões-dentistas que realizam exame radiográfico intrabucal e avaliação das condições para a qualidade do exame. Rev Odontol Unesp. 2003; 32(1):25-9.

15. Miyahara F, Krause D, Haddad D, Costa C, Arita E, Matson M. Estudo da correlação entre a sensibilidade de filmes radiográficos e a variação no tempo de exposição. Rev Inst Ciênc Saúde. 2007; 25(2):153-56.

16. Sarmento VA, Lamberti PLR, Rocha JRM, Beltrão RV, Costa CMA, Pinto RLS et al. Avaliação da influência da sensibilidade do filme radiográfico intrabucal na detecção da lesão periapical artificialmente produzida. Revista Odonto. 2005; 13(25):90-9.

\section{CONFLITO DE INTERESSES}

Os autores declaram não haver conflitos de interesse.

\section{AUTOR PARA CORRESPONDENCIA}

Matheus Henrique Lopes Dominguete

matheus.dominguete@unincor.edu.br

Submetido em 30/05/2018

Aceito em 27/06/2018 